\section{NANOPARTICLE SURFACTANTS}

\section{In a jam}

Science 342, 460-463 (2013)

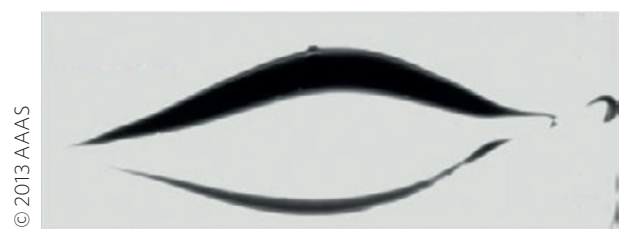

The interface between two different liquids can be used to direct the assembly of nanoparticles. At sufficiently high nanoparticle concentrations such interfaces can lose mobility and start to exhibit solidlike properties, a process that is known as interfacial jamming. Thomas Russell and colleagues the University of Massachusetts Amherst and Tohoku University have now shown that an electric field can be used to manipulate this jamming process and stabilize liquid drops in different nonequilibrium shapes.

The researchers began by forming stable spherical liquid drops. Hydrophilic polystyrene nanoparticles were dispersed in water drops that were suspended in oil. An oleophilic molecule was added to the oil, which could interact with the nanoparticles and form nanoparticle surfactants. The surfactants self-assemble at the water/oil interface and create a disordered, jammed nanoparticle assembly. These spherical drops can then be deformed by applying an external electrical field, which increases the surface area of the drops and allows additional nanoparticle surfactants to form at the interface. When the field is removed, the drop is trapped in this non-equilibrium shape.
The deformation and jamming process can be repeated numerous times, and by applying electric fields in different directions a variety of unusual shapes can be created.

OV

\section{SPIN CALORITRONICS}

\section{Put to the test}

Phys. Rev. Lett. 111, 176601 (2013)

Phys. Rev. Lett. 111, 187201 (2013)

In the spin Seebeck effect, a temperature gradient applied to a ferromagnetic/ non-magnetic bilayer gives rise to a spin current - currents of angular momentum with zero net charge transport - that can be detected by the inverse spin Hall effect in the non-magnetic layer. The spin Seebeck effect has attracted attention because of the possibility of controlling spin currents by thermal flow; however, there is an ongoing debate over the origin and magnitude of the effect due to the potential contamination of the measured spin Seebeck signal by other effects, namely the anomalous Nernst effect and anisotropic magnetoresistance. Two independent research teams have now tried to address this issue and report measurements in yttrium iron garnet/Pt thin-film bilayers and permalloy thin films grown on different substrates.

The two groups use different measurement configurations, longitudinal and transverse, in which a spin current is generated by the spin Seebeck effect either parallel or perpendicular to the thermal gradient, respectively. Using a longitudinal configuration, Sebastian Gönnenwein at the Walther-Meißner-Institut and colleagues in Germany, The Netherlands, China and Japan measure spin pumping, spin Seebeck effect

The chirality, or handedness, of magnetic domain walls can affect the wall propagation direction and speed in current-induced domain wall motion. In thin magnetic films that are in contact with normal metals, the domain wall chirality is induced by the DzyaloshinskiiMoriya interaction at the interface between the two materials. Changes in this interaction can therefore be used to control current-induced domain wall dynamics. Yizheng Wu, Andreas Schmid and colleagues at the Lawrence Berkley National Laboratory, Fudan University and Kyung Hee University have now shown that the chirality of domain walls in $\mathrm{Co} / \mathrm{Ni}$ multilayers can be controlled by engineering the Dzyaloshinskii-Moriya interaction at the interface with the non-magnetic substrate, either Ir or Pt.

The researchers first image the spin structure of chiral Néel-type domain walls by spinpolarized low-energy electron microscopy, and observe the opposite chirality for multilayer stacks on Pt (right-handed) or Ir (left-handed) substrates. Then, by inserting an Ir layer between the $\mathrm{Co} / \mathrm{Ni}$ stack and the Pt substrate, the researchers demonstrate that they can tune the strength of the Dzyaloshinskii-Moriya interaction by varying the thickness of the Ir layer, thus controlling the chirality of the domain walls. By increasing the thickness from 0.6 of a monolayer to 2.5 monolayers and then to 3 monolayers, the researchers observe right-handed chiral Néel walls, achiral Bloch walls and left-handed chiral Néel walls.

and spin Hall magnetoresistance in a series of 20 yttrium iron garnet/Pt samples, and model these effects with the same set of spindependent parameters, demonstrating their spintronic origin. Alternatively, and using a transverse configuration, Christian Back and colleagues at the University of Regensburg and Bielefeld University study the effect of different substrates on the magnitude of the spin Seebeck effect, and find that, regardless of which substrate is used, the contribution of the spin Seebeck effect is negligible compared with those of the anomalous Nernst effect and anisotropic magnetoresistance, in contrast to previous results.

\section{DRUG DELIVERY}

\section{Nanoparticles hitch a ride \\ ACS Nano http://doi.org/p42 (2013)}

The rapid removal of foreign materials by mononuclear phagocytic systems that are located in the spleen and liver limit the ability of nanoparticles to exert their potentially therapeutic effect. One way pathogens can circulate inside the body is by using red blood cells as carriers, an approach known as cellular hitchhiking. The advantage of using red blood cells is that they naturally avoid clearance by mononuclear phagocytic systems. Samir Mitragotri and colleagues at the University of California, Santa Barbara and the University of Pennsylvania have now shown that this strategy can be applied to nanoparticles to increase both their accumulation in body tissues and circulation time.

The researchers incubated polystyrene nanoparticles with red blood cells, injected samples into the tail vein of mice, and observed a marked change in the behaviour of the nanoparticles, compared with free nanoparticles. They detected a seven-fold increase in the accumulation of red blood cell adsorbed-nanoparticles in the lungs and suggest that the nanoparticles are dislodged from the blood cells when being squeezed through the tiny capillaries of the lung vasculature, allowing accumulation in the tissue; the blood cells are able to continue functioning and circulating around the body. Attachment to the blood cells also reduced accumulation in the spleen and liver.

The team improved on this by modifying the surface of the nanoparticle with antiICAM-1, an antibody that can attach to endothelial cells in the lung. This extended the retention of the blood cell adsorbednanoparticles in the lungs from 10 hours to over 24 hours.

Written by Sarah Brown, Elisa De Ranieri and Owain Vaughan. 